\title{
Stability of Solutions of Varying Degenerate Elliptic Equations
}

\section{Li Gongbao $\mathscr{E}$ Olli Martio}

\begin{abstract}
Let $1<p_{0}<\infty, s>p_{0}$ and $p_{i} \rightarrow p_{0}$. For fixed $\psi, \theta \in W^{1, s}(\Omega)$ consider the solution $u_{i}$ to the obstacle problem associated with a second order quasilinear degenerate elliptic equation $\nabla \cdot A_{p_{i}}(x, \nabla u)=0$ with obstacle $\psi$ and boundary values $\theta$. Here $\left|A_{p_{i}}(x, \xi)\right| \approx|\xi|^{p_{i}}$ and the functions $A_{p_{i}}$ have uniformly bounded structure constants. If for a.e. $x \in \Omega, A_{p_{i}}(x, \xi) \rightarrow A_{p_{0}}(x, \xi)$ uniformly on compact subsets of $\mathbb{R}^{n}$, then it is shown that $u_{i} \rightarrow u_{0}$ in $W^{1, t}(\Omega)$ where $u_{0}$ is the corresponding solution to $\nabla \cdot A_{p_{0}}(x, \nabla u)=0$ and $t>p_{0}$ depends on $n, s, p_{0}$, the structure constants, and the regularity of $\Omega$.
\end{abstract}

1. Introduction. Many applications lead to differential equations which are only known approximately. In most cases this concerns the coefficients but in the nonlinear case other parameters could be uncertain as well. In this paper we are interested in the nonlinear degenerate elliptic equation

$$
\nabla \cdot A(x, \nabla u)=0
$$

in divergence form and with measurable coefficients where $|A(x, h)| \approx|h|^{p-1}$. We study the dependence of some classes of solutions to (1.1) on $A$ and this includes the dependence on $p>1$ as well.

A typical situation where the equation (1.1) has a unique solution is the Dirichlet boundary value problem and the obstacle problem. We keep the boundary values and the obstacle fixed; the dependence on this data is studied in [LM2] and the combination of the results in [LM2] and the results here give a rather complete description on the stability of the solutions with respect to all data. Let $p_{i} \rightarrow p_{0} \in(1, \infty)$ and $A_{p_{i}} \rightarrow A_{p_{0}}$ in the sense specified in Section 2. Consider the solution $u_{i}$ to the equation $\nabla \cdot A_{p_{i}}(x, \nabla u)=0, i=0,1, \ldots$, with respect to the given data in a bounded domain $\Omega$ of $\mathbb{R}^{n}$. Our result says that $u_{i} \rightarrow u_{0}$ in the Sobolev space $W^{1, p_{0}+\varepsilon}(\Omega)$ for some $\varepsilon>0$ whose dependence on $A_{i}, i=0,1$, 
$\ldots$, and the data will also become clear. The proof consists of two parts. First we show that $u_{i} \rightarrow \bar{u}$ in $W^{1, p_{0}+\varepsilon}(\Omega)$ and then that $\bar{u}=u_{0}$. It is rather easy to see that $\bar{u}$ is a local solution or a supersolution to $\nabla \cdot A_{p_{0}}(x, \nabla u)=0$. For $\bar{u}=u_{0}$ the cases $p_{i} \nearrow p_{0}$ and $p_{i} \searrow p_{0}$ impose different difficulties.

The higher integrability of the gradients of solutions and supersolutions to (1.1) plays an essential role. Since we are interested in the global situation, the regularity of $\Omega$ as well as the regularity of the boundary data and the obstacle are important. These questions have been studied for a fixed equation in $[\mathrm{G}$, HKM, I, and LM1], see also references therein. We employ uniform estimates from the previous works.

The behavior of extremals of the variational inegral

$$
\int_{\Omega}|\nabla u|^{p} d x
$$

with respect to $p$ has been studied in $[\mathrm{Li}]$. This corresponds to the $p$-harmonic case $A_{p}(x, h)=|h|^{p-2} h$. The obstacle problem, essential to the theory of supersolutions, has not been considered previously.

Section 2 contains the assumptions and the main results. Section 3 is devoted to the preliminary lemmas and the main result is proved in Section 4.

2. Main results. To formulate our results we need some notation. Let $1<p<\infty, 0<\alpha \leq \beta<\infty$, and $\Omega$ a bounded open set in $\mathbb{R}^{n}$. We consider the second order quasilinear degenerate elliptic equation

$$
\nabla \cdot A_{p}(x, \nabla u)=0
$$

where the mapping $A_{p}: \Omega \times \mathbb{R}^{n} \rightarrow \mathbb{R}^{n}$ satisfies the assumptions:

(a) the mapping $x \mapsto A_{p}(x, \xi)$ is measurable for all $\xi \in \mathbb{R}^{n}$.

For all $\xi \in \mathbb{R}^{n}$ and a.e. $x \in \Omega$ :

(b) $A_{p}(x, \xi) \cdot \xi \geq \alpha|\xi|^{p}$,

(c) $\left|A_{p}(x, \xi)\right| \leq \beta|\xi|^{p-1}$,

(d) $\left(A_{p}\left(x, \xi_{1}\right)-A_{p}\left(x, \xi_{2}\right)\right) \cdot\left(\xi_{1}-\xi_{2}\right)>0$ whenever $\xi_{1} \neq \xi_{2}$.

In the sequel we will consider operators $A_{p}$ with varying $p$. Note that we shall keep $\alpha$ and $\beta$ fixed.

For $1<p<\infty$ we let $W^{1, p}(\Omega)$ denote the Sobolev space of functions $u \in L^{p}(\Omega)$ whose distributional gradient $\nabla u$ belongs to $L^{p}(\Omega)$. In $W^{1, p}(\Omega)$ we use the standard norm

$$
\|u\|_{1, p}=\|u\|_{W^{1, p}(\Omega)}=\|u\|_{p}+\|\nabla u\|_{p}
$$

where $\|u\|_{p}=\|u\|_{L^{p}(\Omega)}$. The space $W_{0}^{1, p}(\Omega)$ is the closure of $C_{0}^{\infty}(\Omega)$ in $W^{1, p}(\Omega)$.

Next we recall the obstacle problem associated with (2.1). 
Let $\theta \in W^{1, p}(\Omega)$ and let $\psi: \Omega \rightarrow \mathbb{R} \cup\{ \pm \infty\}$ be measurable. Write

$$
\mathcal{K}_{\psi, \theta}^{p}=\left\{u \in W^{1, p}(\Omega): u-\theta \in W_{0}^{1, p}(\Omega), u \geq \psi \text { a.e. }\right\}
$$

A function $u \in W^{1, p}(\Omega)$ is a solution to the $\mathcal{K}_{\psi, \theta}^{p}$-obstacle problem of the equation (2.1) if $u \in \mathcal{K}_{\psi, \theta}^{p}$ and if

$$
\int_{\Omega} A_{p}(x, \nabla u(x)) \cdot \nabla(v-u)(x) d x \geq 0
$$

for all $v \in \mathcal{K}_{\psi, \theta}^{p}$. If $\mathcal{K}_{\psi, \theta}^{p} \neq \varnothing$, then there is a unique solution to the $\mathcal{K}_{\psi, \theta}^{p}$-obstacle problem, see [HKM, pp. 60-61].

Let $\Omega$ be a bounded domain in $\mathbb{R}^{n}$. We say that $\Omega^{c}$ is thick if for each cube $Q(r)$ with $Q(3 r / 2) \cap \Omega^{c} \neq \varnothing$ we have

$$
\left|\Omega^{c} \cap Q(2 r)\right| \geq \mu|Q(2 r)|
$$

where $\mu>0$ is independent of the cube $Q(r)$. Here and in the sequel $Q(\lambda r)$ denotes the cube parallel to $Q(r)$ with the same center and side length $\lambda r$. We let $|A|$ stand for the $n$-measure of $A \subset \mathbb{R}^{n}$.

Let $1<p_{0}<\infty$ and $s>p_{0}$. Suppose that $\psi, \theta \in W^{1, s}(\Omega)$ where $\Omega$ is a bounded domain satisfying $(2.3)$ and $\mathcal{K}_{\psi, \theta}^{s} \neq \varnothing$. Next suppose that $\left(p_{i}\right)$ is a sequence such that $p_{i} \rightarrow p_{0}$ and that for each $i=0,1, \ldots$ there is an operator $A_{i}=A_{p_{i}}$ satisfying (a)-(d) for $p=p_{i}$ and for a.e. $x \in \Omega$

$$
A_{i}(x, \xi) \rightarrow A_{0}(x, \xi)
$$

uniformly on compact subsets of $\mathbb{R}^{n}$. Now for $i=0$ and for large $i$, in fact for $p_{i} \leq s$, there is a unique solution $u_{i}$ to the $\mathcal{K}_{\psi, \theta}^{p_{i}}$-obstacle problem for the equation $\nabla \cdot A_{i}(x, \nabla u)=0$. Our main theorem now takes the following form.

2.5. Theorem. There is $t>p_{0}$ such that $u_{i} \rightarrow u_{0}$ in $W^{1, t}(\Omega)$. Moreover, $t$ depends only on $n, p_{0}, s, \alpha, \beta$, and $\mu$, where $\alpha$ and $\beta$ are as in (b), (c), and $\mu$ as in (2.3).

\subsection{Remarks.}

(a) It is possible to consider the cases $p_{i} \nearrow p_{0}$ and $p_{i} \searrow p_{0}$ separately. In the case $p_{i} \nearrow p_{0}$ there is no need to assume that $\psi, \theta \in W^{1, s}(\Omega)$ for $s>p_{0}$. However, then it is not possible to achieve a global convergence in $W^{1, t}(\Omega)$, $t>p_{0}$. For simplicity, we consider both cases simultaneously.

(b) The condition (2.3) for $\Omega^{c}$ is too strong. In fact, this condition is only used for the optimal Sobolev inequality and for a conclusion that a certain function belongs to $W_{0}^{1, p_{0}}(\Omega)$, see Lemma 3.25. For these purposes some capacity thickness conditions for $\Omega^{c}$ suffice. These conditions depend on the 
exponent $p$, and the condition (2.3) has been chosen because it provides a simple sufficient condition independent of $p>1$. Note that (2.3) is satisfied if $\partial \Omega$ is smooth.

A much simplified version of the proof for Theorem 2.5 produces a stability result for solutions of the equations $\nabla \cdot A_{i}(x, \nabla u)=0$. We recall the necessary concepts. Let $\theta \in W^{1, p}(\Omega)$. A function $u \in W^{1, p}(\Omega)$ is called the solution of $\nabla \cdot A_{p}(x, \nabla u)=0$ with boundary value $\theta$ if $u-\theta \in W_{0}^{1, p}(\Omega)$ and if

$$
\int_{\Omega} A_{p}(x, \nabla u(x)) \cdot \nabla \varphi(x) d x=0
$$

for all $\varphi \in C_{0}^{\infty}(\Omega)$. If $A_{p}$ satisfies (a)-(d), then there is a unique solution $u$ with boundary values $\theta$, see $[\mathrm{HKM}]$.

To formulate the result let $p_{0}>1$ and $p_{i} \rightarrow p_{0}$. Suppose that $\theta \in W^{1, s}(\Omega)$, $s>p_{0}$, where $\Omega$ satisfies (2.3). Let $u_{i}$ be the solution of $\nabla \cdot A_{p_{i}}(x, \nabla u)=0$ with boundary values $\theta$; we assume that $A_{i}=A_{p_{i}}$ satisfies (a)-(d), $i=0,1, \ldots$ and that (2.4) holds.

2.8. Theorem. There is $t>p_{0}$ such that $u_{i} \rightarrow u_{0}$ in $W^{1, t}(\Omega)$. The constant $t$ depends on the same parameters as in Theorem 2.5.

We shall not prove Theorem 2.8. It is enough to note that in this case the obstacle $\psi$ can be completely omitted from considerations and as a test function $\varphi$ in (2.7) it is possible to use any $\varphi$ in $W_{0}^{1, p}(\Omega)$.

2.9. Remark. The method of Theorem 2.5 can be employed to produce other stability results as well. In particular, local stability results are much easier since a local solution or supersolution for $\nabla \cdot A_{p}(x, \nabla u)=0$ is not uniquely defined and hence a stability result of this kind would only state that a sequence of solutions or supersolutions $\left(u_{i}\right)$ for $\nabla \cdot A_{p_{i}}\left(x, \nabla_{n}\right)=0$ converges to some solution or supersolution for $\nabla \cdot A_{p_{0}}(x, \nabla u)=0$.

3. Preliminary results: Higher integrability, zero boundary values. In this section we present a global higher inequality result for solutions to the obstacle problem associated with the equation $\nabla \cdot A(x, \nabla u)=0$. There is an abundance of results of this kind. However, global results are more difficult to obtain and we need results which are uniform with respect to the exponent $p>1$. Our result is an extension of [LM1, Theorem B] in this sense. The most convenient real analytic lemma for our purposes is [I, Proposition 6.1], which gives explicit bounds for higher integrability exponents. We have also taken some care to collect all the other estimates in such a form that the dependence on $p$ is uniform.

Our second preliminary result gives a sufficient condition such that each function $u \in W^{1, p}(\Omega)$ with $u \in W_{0}^{1, t}(\Omega), t<p$, also belongs to $W_{0}^{1, p}(\Omega)$. Here we use a recent result from $[\mathrm{KinM}]$. 
We shall use the following convention. Suppose that the parameter $t_{i}$ belongs to an interval $\Delta_{i}, i=1,2, \ldots$ We say that a constant $C=C\left(t_{1}, t_{2}, \ldots\right)$ satisfies $C<\infty$ uniformly for $t_{1} \in \Delta_{1}$, say, if for each $t_{2}, t_{3}, \ldots$ and for each compact subset $F \subset \Delta_{1}$ there is $c_{0}<\infty$ such that $C \leq c_{0}$ for all $t_{1} \in F$. Obviously this definition extends to several parameters and to the case $C>0$ as well. Various constants, denoted usually by $C$, may differ even on the same line.

For the higher integrability result in the obstacle problems let $\Omega$ be a bounded domain with the property (2.3) and let $A=A_{p}$ satisfy (a)-(d) in $\Omega$. Suppose that $\psi, \theta \in W^{1, p}(\Omega)$ and that $u$ is a solution to the $\mathcal{K}_{\psi, \theta}^{p}$-obstacle problem; the existence requires that $\mathcal{K}_{\psi, \theta}^{p} \neq \varnothing$. We let $d(\Omega)$ denote the diameter of $\Omega$.

3.1. Lemma. There exsits a constant $\varepsilon_{0}=\varepsilon_{0}(n, p, \alpha, \beta, \mu)>0$ uniformly for $p>1$ such that for each $\varepsilon \in\left[0, \varepsilon_{0}\right]$

$$
\int_{\Omega}|\nabla u|^{p+\varepsilon} d x \leq C\left[\left(\int_{\Omega}|\nabla u|^{p} d x\right)^{1+\varepsilon / p}+\int_{\Omega}|\nabla \psi|^{p+\varepsilon} d x+\int_{\Omega}|\nabla \theta|^{p+\varepsilon} d x\right]
$$

where $C=C(n, p, \alpha, \beta, \mu, d(\Omega))<\infty$ uniformly for $p>1$.

3.3. Remark. The proof for Lemma 3.1 shows that the constants $\varepsilon_{0}$ and $C$ satisfy $\varepsilon_{0}>0$ and $C<\infty$ uniformly for $p>1,0<\alpha \leq \beta<\infty, \mu \in(0,1]$ and $d(\Omega)>0$ and not for $p>1$ only. Note that the right hand side of (3.2) is $\infty$, if $\nabla \psi$ or $\nabla \theta$ does not belong to $L^{p+\varepsilon}$. Lemma 3.1 will be used when $\psi$ and $\theta$ belong to the higher Sobolev space.

For the proof of Lemma 3.1 various Sobolev and Poincaré type inequalities are needed. We will employ the following Poincaré inequality for a function $u \in W^{1, p}(Q), p>1$, in a cube $Q \subset \mathbb{R}^{n} ;$

$$
\left(\int_{Q}\left|u-u_{Q}\right|^{q} d x\right)^{1 / q} \leq C\left(\int_{Q}|\nabla u|^{p} d x\right)^{1 / p}
$$

where $u_{Q}$ is the mean value of $u$ in $Q$ and

$$
C=c(n)\left(\frac{1-\frac{1}{p}+\frac{1}{q}}{\frac{1}{n}-\frac{1}{p}+\frac{1}{q}}\right)^{1-1 / p+1 / q}|Q|^{1 / n-1 / p+1 / q}
$$

for $q \geq p$ and $q<n p /(n-p)$ for $p<n$. For $p<n$ and $q=n p /(n-p)$ the inequality (3.4) holds with

$$
C=c(n)\left(\frac{p}{n-p}\right)\left(\frac{p}{p-1}\right)^{(n-p) / n p}
$$


The inequality (3.4) follows from the potential estimate [GT, Lemma 7.16, p. 162] together with [GT, Lemma 7.12, p. 159]; for the case $q=n p /(n-p)$ the aforementioned potential estimate with $[\mathrm{Z}$, Theorem 2.8.4] yields the result.

If $u \in W_{0}^{1, p}(\Omega), p \geq 1$, then

$$
\|u\|_{p} \leq c(n)\|\nabla u\|_{p}
$$

see [GT, 7.44]. In fact, the method used in the proof of Lemma 3.8 below provides a simple and explicit approach to (3.4) and (3.7).

3.8. Lemma. Suppose that $\Omega$ is a bounded domain with the property (2.3) and that $q \geq 1$. Let $Q(r)$ be a cube with side length $r$ such that $Q(3 / 2 r) \cap \Omega^{c} \neq \varnothing$. Then for each $u \in W^{1, q}(Q(2 r))$

$$
\int_{Q(2 r)}|u|^{q} d x \leq C r^{q} \int_{Q(2 r)}|\nabla u|^{q} d x
$$

provided that $u=0$ on $Q(2 r) \cap \Omega^{c}$. The constant $C=C(n, q, \mu)$ satisfies $C<\infty$ uniformly for $q \geq 1$.

3.10. Remark. Lemma 3.8 is far from the best possible. The sharp versions of (3.9) assume that $u=0$ on $K \cap Q(2 r)$ where $K$ has positive $(1, q)$ capacity only. In this case the function $u$ is defined in the capacity sense, see [AH, p. 231] for the history of the problem. The inequality (3.9) also appears for $q=1$ in [LU, Lemma 3.4, p. 76]. However, we need an explicit bound for the constant $C$ in order to take the dependence on $q$ into account. Hence we present a simple proof for Lemma 3.8.

Proof for Lemma 3.8. Write $Q=Q(2 r)=\left\{x: 0<x_{i}<2 r\right\}$ and choose a sequence of functions $u_{i} \in C^{1}(Q)$ such that $u_{i} \rightarrow u$ a.e. and $u_{i} \rightarrow u$ in $W^{1, p}(Q)$. Fix $\varepsilon>0$ and let

$$
A_{i}=\left\{x \in Q:\left|u_{i}(x)\right|<\varepsilon\right\} .
$$

Since $\bigcup_{j=1}^{\infty} \bigcap_{i=j}^{\infty} A_{i}$ contains almost all the points of the set $A=\{u(x)=0\}$ and since $\bigcap_{i=j}^{\infty} A_{i} \subset \bigcap_{i=j+1}^{\infty} A_{i}$,

$$
|A| \leq\left|\bigcup_{j=1}^{\infty} \bigcap_{i=j}^{\infty} A_{i}\right|=\lim _{j \rightarrow \infty}\left|\bigcap_{i=j}^{\infty} A_{i}\right| \leq \liminf _{j \rightarrow \infty}\left|A_{j}\right| .
$$

Let $x=\left(x_{1}, \ldots, x_{n}\right), y=\left(y_{1}, \ldots, y_{n}\right)$ be points in $Q$. Integrating the partial derivatives of $u_{j}$ along a path consisting of line segments parallel to coordinate 
axis we obtain

$$
\begin{aligned}
\left|u_{j}(x)\right|-\left|u_{j}(y)\right| & \leq\left|u_{j}(x)-u_{j}(y)\right| \\
& \leq \sum_{i=1}^{n} \int_{0}^{2 r}\left|\nabla u_{j}\left(y_{1}, \ldots, y_{i-1}, s, x_{i+1}, \ldots, x_{n}\right)\right| d s
\end{aligned}
$$

The Hölder inequality yields

$$
\left|u_{j}(x)\right|^{q} \leq(n+1)^{q-1}\left[\sum_{i=1}^{n}(2 r)^{q-1} \int_{0}^{2 r}\left|\nabla u_{j}\left(y_{1}, \ldots, x_{n}\right)\right|^{q} d s+\left|u_{j}(y)\right|^{q}\right] .
$$

Integrating with respect to $y \in A_{j}$ and then with respect to $x \in Q$ we have by the Fubini theorem

$$
\left|A_{j}\right| \int_{Q}\left|u_{j}\right|^{q} d x \leq(n+1)^{q-1}\left[\sum_{i=1}^{n}(2 r)^{q-1+n+1} \int_{Q}\left|\nabla u_{j}\right|^{q} d x+\varepsilon^{q}\left|A_{j}\right||Q|\right] .
$$

Hence

$$
\left|A_{j}\right| \int_{Q}\left|u_{j}\right|^{q} d x \leq(n+1)^{q-1}\left[n(2 r)^{q+n} \int_{Q}\left|\nabla u_{j}\right|^{q} d x+\varepsilon^{q}\left|A_{j}\right||Q|\right]
$$

and thus by $(2.3)$

$$
\begin{aligned}
\int_{Q}\left|u_{j}\right|^{q} d x & \leq(n+1)^{q-1}\left[n \frac{|Q|}{\left|A_{j}\right|}(2 r)^{q} \int_{Q}\left|\nabla u_{j}\right|^{q} d x+\varepsilon^{q}|Q|\right] \\
& \leq(n+1)^{q-1}\left[\frac{n}{\mu}(2 r)^{q} \int_{Q}\left|\nabla u_{j}\right|^{q} d x+\varepsilon^{q}|Q|\right] .
\end{aligned}
$$

Letting $j \rightarrow \infty$ and then $\varepsilon \rightarrow 0$ we obtain

$$
\int_{Q}|u|^{q} d x \leq \frac{(n+1)^{q}}{\mu}(2 r)^{q} \int_{Q}|\nabla u|^{q} d x
$$

as required.

Now the previous lemma and (3.4) easily provide the following result:

3.11. Lemma. Let $Q(r)$ and $\Omega$ be as in Lemma 3.8 with $r \leq 2 d(\Omega) \leq 2$. If $u \in W^{1, p}(Q(2 r)), p>1$, and if $u=0$ in $Q(2 r) \cap \Omega^{c}$, then

$$
\left(\int_{Q(2 r)}|u|^{p} d x\right)^{1 / p} \leq C\left(\int_{Q(2 r)}|\nabla u|^{q} d x\right)^{1 / q}
$$


where $q=p n /(n+p)$ and the constant $C=C(n, p, \mu)$ satisfies $C<\infty$ uniformly for $p>1$.

Proof. Since $q \leq p$, Lemma 3.8 yields

$$
\int_{Q(2 r)}|u|^{q} d x \leq C r^{q} \int_{Q(2 r)}|\nabla u|^{q} d x .
$$

Now let $c_{u}$ be the mean value of $u$ in $Q(2 r)$. By (3.4)

$$
\left(\int_{Q(2 r)}\left|u-c_{u}\right|^{p} d x\right)^{1 / p} \leq C\left(\int_{Q(2 r)}|\nabla u|^{q} d x\right)^{1 / q}
$$

where the constant $C$ in $(3.5)$ can be estimated from above in terms of $d(\Omega)$ because $r \leq 4 d(\Omega)$. From (3.13) and (3.14) we obtain

$$
\begin{aligned}
\left(\int_{Q(2 r)}|u|^{p} d x\right)^{1 / p} & \leq\left(\int_{Q(2 r)}\left|u-c_{u}\right|^{p} d x\right)^{1 / p}+|Q(2 r)|^{1 / p}\left|c_{u}\right| \\
& \leq C\left(\int_{Q(2 r)}|\nabla u|^{q} d x\right)^{1 / q}+|Q(2 r)|^{-1 / n}\left(\int_{Q(2 r)}|u|^{q} d x\right)^{1 / q} \\
& \leq C\left(\int_{Q(2 r)}|\nabla u|^{q} d x\right)^{1 / q}
\end{aligned}
$$

The inspection of the constant $C$ on the last line easily gives the desired property. The lemma follows.

Our last lemma is the important higher integrability condition. The formulation due to Iwaniec [I, Proposition 6.1] is convenient for our purposes because the constants have been computed explicitely.

3.15. Lemma $([\boldsymbol{I}$, Proposition 6.1 $])$. Let $Q_{0}=Q_{0}(l)$ be a cube in $\mathbb{R}^{n}$ and let $g, h \in L^{q}\left(Q_{0}\right), 1<q<\infty$, be nonnegative functions satisfying

$$
\left(f_{Q(r)} g^{q} d x\right)^{1 / q} \leq K f_{Q(2 r)} g d x+\left(f_{Q(2 r)} h^{q} d x\right)^{1 / q}
$$

for all cubes $Q(r) \subset Q(2 r) \subset Q_{0}$ parallel to $Q_{0}$. Then for each $\sigma \in(0,1)$ and

$$
q \leq s<q+\frac{q-1}{10^{n-q 4^{n}} K^{q}}
$$


we have

$$
\left(f_{Q_{0}(\sigma l)} g^{s} d x\right)^{1 / 2} \leq C\left[\left(f_{Q_{0}} g^{q} d x\right)^{1 / q}+\left(f_{Q_{0}} h^{s} d x\right)^{1 / s}\right]
$$

where

$$
C=\frac{100^{n}}{\sigma^{n / s}(1-\sigma)^{n / q}} .
$$

Proof for Lemma 3.1. We prove the lemma for $d(\Omega) \leq 1$ with the constant $C$ in (3.2) independent of $d(\Omega)$. For $d(\Omega)>1$ an application of a dilation map produces the required result with the same $\varepsilon_{0}$ and with a constant $C$ depending on $d(\Omega)$ as well.

Pick a cube $Q_{0}=Q_{0}\left(2 r_{0}\right)$ such that $\Omega \subset Q_{0}\left(r_{0}\right)$ and $r_{0} \leq d(\Omega) \leq 1$. Next take an arbitrary cube $Q(2 r)$ in $Q_{0}$. There are two possibilities

(i) $Q(3 r / 2) \subset \Omega$ or

(ii) $Q(3 r / 2) \cap \Omega^{c} \neq \varnothing$.

In the case (i) we can use the previous estimates just as in [LM1, Proof for Theorem B, p. 31] to obtain

$$
\begin{aligned}
& f_{Q(r)}|\nabla u|^{p} d x \\
& \quad \leq C\left[\left(f_{Q(3 r / 2)}|\nabla u|^{n p /(n+p)} d x\right)^{(p+n) / n}+f_{Q(3 r / 2)}|\nabla \psi|^{p} d x\right]
\end{aligned}
$$

where $C=C(n, p, \alpha, \beta)<\infty$ uniformly for $p>1$.

In the case (ii) we can proceed similarly as in [LM1, Proof for Theorem B, pp. 31-32] and obtain

$$
\begin{aligned}
& \int_{\Omega} \varphi^{p}|\nabla u|^{p} d x \\
& \quad \leq C\left[r^{-p}\left(\int_{Q(2 r) \cap \Omega}|\nabla u|^{n p /(n+p)} d x\right)^{(p+n) / n}+\int_{Q(2 r) \cap \Omega}|\nabla \theta|^{p} d x\right]
\end{aligned}
$$

where $C=C(n, p, \alpha, \beta, \mu)<\infty$ uniformly for $p>1$ and where $\varphi$ is a cut off function such that $\varphi \in C_{0}^{\infty}(Q(2 r)), 0 \leq \varphi \leq 1,|\nabla \varphi| \leq C(n) / r$ and $\varphi=1$ on $Q(r)$.

For the inequalities (3.18) and (3.19) we have employed the uniform estimates (3.4) and (3.12).

To complete the proof for the inequality (3.2) we let

$$
g=|\nabla u|^{n p /(n+p)}, f=(|\nabla \theta|+|\nabla \psi|)^{n p /(n+p)}
$$


in $\Omega$ and $g=0=f$ in $\Omega^{c}$. By (3.18) and (3.19) for any $Q(2 r) \subset Q_{0}$ we have

$$
f_{Q(r)} g^{q} d x \leq C_{1}\left[\left(f_{Q(2 r)} g d x\right)^{q}+f_{Q(2 r)} f^{q} d x\right]
$$

where $q=(p+n) / n$ and $C_{1}=C_{1}(n, p, \alpha, \beta, \mu)<\infty$ uniformly for $p>1$; this is also true for all the constants $C$ which will appear in the rest of the proof. Rewriting (3.20) in the form

$$
\left(f_{Q(r)} g^{q} d x\right)^{1 / q} \leq C_{1}^{1 / q} f_{Q(2 r)} g d x+\left(f_{Q(2 r)}(C f)^{q} d x\right)^{1 / q}
$$

we obtain from Lemma 3.15 with $\sigma=1 / 2$ that

$$
\left(f_{Q_{0}\left(r_{0}\right)} g^{s} d x\right)^{1 / s} \leq C\left[\left(f_{Q_{0}} g^{q} d x\right)^{1 / q}+\left(f_{Q_{0}} f^{s} d x\right)^{1 / s}\right]
$$

for any $s \in\left[q, q+\varepsilon_{1}\right)$ where $\varepsilon_{1}=\varepsilon_{1}(n, p, \alpha, \beta, \mu)>0$ uniformly for $p>1$. Writing (3.22) with the original exponents we obtain

$$
\begin{aligned}
& \int_{\Omega}|\nabla u|^{n p s /(n+p)} d x \\
& \quad \leq C\left[\left(\int_{\Omega}|\nabla u|^{n p q /(n+p)} d x\right)^{s / q}+\int_{\Omega}(|\nabla \theta|+|\nabla \psi|)^{n p s /(n+p)} d x\right] .
\end{aligned}
$$

We fix $\varepsilon_{0}=n p \varepsilon_{1} / 2(n+p)>0$ and let $\varepsilon \in\left[0, \varepsilon_{0}\right]$. For

$$
s=\frac{n+p}{n}\left(1+\frac{\varepsilon}{p}\right) \leq q+\varepsilon_{1} / 2
$$

(3.23) yields

$$
\int_{\Omega}|\nabla u|^{p+\varepsilon} d x \leq C\left[\left(\int_{\Omega}|\nabla u|^{p} d x\right)^{1+\varepsilon / p}+\int_{\Omega}(|\nabla \theta|+|\nabla \psi|)^{p+\varepsilon} d x\right]
$$

and this is the desired inequality $(3.2)$ for the case $d(\Omega) \leq 1$.

Our last lemma concerns functions with zero boundary values in the Sobolev sense. The formulation is more general than what is needed in Section 4 . 
3.25. Lemma. Suppose that a bounded open set $\Omega$ satisfies (2.3). Let $p_{i}>1$ and $u_{i} \in W^{1, p_{i}}(\Omega), i=0,1, \ldots$, and suppose that $p_{i} \rightarrow p_{0}$ and $u_{i} \rightarrow u_{0}$ a.e. in $\Omega$. If $\theta \in W^{1, p_{0}+\varepsilon}(\Omega), \varepsilon>0$, and if $u_{i}-\theta \in W_{0}^{1, p_{i}}(\Omega)$ with

$$
\int_{\Omega}\left|\nabla\left(u_{i}-\theta\right)\right|^{p_{i}} d x \leq M
$$

$i=1,2, \ldots$, where $M<\infty$ is independent of $i$, then $u_{0}-\theta \in W_{0}^{1, p_{0}}(\Omega)$.

\subsection{Remarks.}

(a) The lemma is trivial if $p_{i} \searrow p_{0}$. In the case $p_{i} \nearrow p_{0}$ the condition $\theta \in$ $W^{1, p_{0}+\varepsilon}(\Omega)$ can be replaced by $\theta \in W^{1, p_{0}}(\Omega)$.

(b) We employ Lemma 3.25 in the situation where $u_{i} \in W^{1, p_{0}+\varepsilon}(\Omega), i=1,2$, $\ldots$, with $\left\|\nabla u_{i}\right\|_{p_{0}+\varepsilon} \leq C$. Then (3.26) is clear but the conclusion of the lemma is not trivial for $p_{i} \nearrow p_{0}$.

Proof for Lemma 3.25. Since $\Omega$ satisfies (2.3), $\Omega$ also satisfies the uniform Hardy inequality

$$
\int_{\Omega}\left(\frac{|u(x)|}{d(x, \partial \Omega)}\right)^{p} d x \leq C \int_{\Omega}|\nabla u(x)|^{p} d x
$$

for all $u \in W_{0}^{1, p}(\Omega)$ where $C=C(n, p, \mu)<\infty$ uniformly for $p>1$. This is due to the fact that condition (2.3) gives a uniform lower estimate for the $(1, p)$-capacity of $B(x, r) \cap \Omega^{c}$ in terms of the $(1, p)$-capacity of $B(x, r)$ at each boundary point $x \in \partial \Omega$. See [Le, Mi, and KinM].

From (3.28) and (3.26) we obtain

$$
\int_{\Omega}\left(\frac{\left|u_{i}-\theta\right|}{d(x, \partial \Omega)}\right)^{p_{i}} d x \leq C \int_{\Omega}\left|\nabla\left(u_{i}-\theta\right)\right|^{p} d x
$$

and the Fatou Lemma gives

$$
\int_{\Omega}\left(\frac{\left|u_{0}-\theta\right|}{d(x, \partial \Omega)}\right)^{p_{0}} d x \leq \liminf _{i \rightarrow \infty} \int_{\Omega}\left(\frac{\left|u_{i}-\theta\right|}{d(x, \partial \Omega)}\right)^{p_{i}} d x \leq C M .
$$

Hence

$$
\frac{u_{0}-\theta}{d(x, \partial \Omega)} \in L^{p_{0}}(\Omega)
$$

and since $u_{0}-\theta \in W^{1, p_{0}}(\Omega),\left[\mathrm{KinM}\right.$, Theorem 3.13] implies that $u_{0}-\theta \in$ $W_{0}^{1, p_{0}}(\Omega)$ as required. 
4. Proof for Theorem 2.5. Since $p_{i} \rightarrow p_{0}$ and since $s>p_{0}$, we may assume that $p_{i}<s$ for all $i$. Then $\max (\theta, \psi) \in \mathcal{K}_{\psi, \theta}^{p_{i}}$ and hence there is a unique solution $u_{i}, i=0,1, \ldots$, to the $\mathcal{K}_{\psi, \theta}^{p_{i}}$-obstacle problem for the equation $\nabla \cdot A_{p_{i}}(x, \nabla u)=0$. We write

$$
A_{i}=A_{p_{i}}, i=0,1, \ldots .
$$

The proof is now carried out in several steps. The constant $t>p_{0}$ will always depend on the parameters of the theorem although it is necessary to make it smaller in certain steps of the proof. We first show that

$$
\int_{\Omega}\left|\nabla u_{i}\right|^{p_{i}} d x \leq C
$$

where $C<\infty$ is independent of $i=0,1, \ldots$ Replacing $\theta$ by $\max (\theta, \psi)$ we may assume that $\theta \in \mathcal{K}_{\psi, \theta}^{p_{i}}$ and hence

$$
\int_{\Omega} A_{i}\left(x, \nabla u_{i}\right) \cdot \nabla\left(\theta-u_{i}\right) d x \geq 0
$$

This yields

$$
\begin{aligned}
\alpha \int_{\Omega}\left|\nabla u_{i}\right|^{p_{i}} d x & \leq \int_{\Omega} A_{i}\left(x \cdot \nabla u_{i}\right) \cdot \nabla u_{i} d x \\
& \leq \int_{\Omega} A_{i}\left(x, \nabla u_{i}\right) \cdot \nabla \theta d x \leq \beta \int_{\Omega}\left|\nabla u_{i}\right|^{p_{i}-1}|\nabla \theta| d x \\
& \leq \beta\left(\int_{\Omega}\left|\nabla u_{i}\right|^{p_{i}} d x\right)^{\left(p_{i}-1\right) / p_{i}}\left(\int_{\Omega}|\nabla \theta|^{p_{i}} d x\right)^{1 / p_{i}}
\end{aligned}
$$

and hence

$$
\left\|\nabla u_{i}\right\|_{p_{i}} \leq \frac{\beta}{\alpha}\|\nabla \theta\|_{p_{i}}
$$

By the Hölder inequality this gives

$$
\int_{\Omega}\left|\nabla u_{i}\right|^{p_{i}} d x \leq\left(\frac{\beta}{\alpha}\right)^{s} \max (1,|\Omega|) \int_{\Omega}|\nabla \theta|^{s} d x=C,
$$

and (4.1) follows.

Next we employ the higher integrability estimate of Lemma 3.1 and find $t \in\left(p_{0}, s\right]$ and $C<\infty$ such that

$$
\int_{\Omega}\left|\nabla u_{i}\right|^{t} d x \leq C\left[\left(\int_{\Omega}\left|\nabla u_{i}\right|^{p_{i}} d x\right)^{1+\left(t-p_{i}\right) / p_{i}}+\int_{\Omega}|\nabla \psi|^{t} d x+\int_{\Omega}|\nabla \theta|^{t} d x\right] .
$$


This estimate holds for $i=0$ and for large $i$; hence we may assume that it holds for all $i$. Moreover, by the uniformity of the higher integrability estimate the constants $C$ and $t$ are independent of $i$. From (4.1) and (4.2) we obtain

$$
\int_{\Omega}\left|\nabla u_{i}\right|^{t} d x \leq C, \quad i=0,1, \ldots
$$

where $C<\infty$ is independent of $i$.

The function $u_{i}-\theta$ belongs to $W_{0}^{1, p_{i}}(\Omega)$ and hence by the Sobolev imbedding theorem

$$
\left\|u_{i}-\theta\right\|_{p_{i}^{*}} \leq C\left\|\nabla\left(u_{i}-\theta\right)\right\|_{p_{i}}
$$

where $p_{i}^{*}$ is the Sobolev conjugate exponent $p_{i} n /\left(n-p_{i}\right)$ if $p_{i}<n$ and $p_{i}^{*}=t$ if $p_{i} \geq n$. The constant $C$ is again independent of $i$. Choosing $t \in\left(p_{0}, p_{0}^{*}\right)$ we thus obtain from (4.3) and (4.4)

$$
\left\|u_{i}\right\|_{t} \leq C\left\|u_{i}\right\|_{p_{i}^{*}} \leq C\left[\left\|u_{i}-\theta\right\|_{p_{i}^{*}}+\|\theta\|_{p_{i}^{*}}\right] \leq C\left[\left\|\nabla\left(u_{i}-\theta\right)\right\|_{p_{i}}+\|\theta\|_{p_{i}^{*}}\right]
$$

and this holds for $i=0$ and for large $i$. Now (4.3) and (4.4) yield

$$
\left\|u_{i}\right\|_{1, t} \leq C
$$

where $C<\infty$ and $t \in\left[p_{0}, s\right]$ are independent of $i$; the estimate (4.6) holds for $i=0$ and for large $i$.

Since the Sobolev norms in (4.6) for $i=1,2, \ldots$ are bounded, for some subsequence of $u_{i}$ we have that $u_{i} \rightarrow \bar{u}$ weakly in $W^{1, t}(\Omega)$ and that

$$
\begin{aligned}
& u_{i} \rightarrow \bar{u} \quad \text { a.e. } \\
& u_{i} \rightarrow \bar{u} \text { in } L^{t}(\Omega) .
\end{aligned}
$$

Note that in the sequel we shall prove that $\bar{u}=u_{0}$ and hence this convergence holds for the whole sequence, not only for some subsequence of the original sequence.

Now $u_{i} \geq \psi$ and hence by (4.7) $\bar{u} \geq \psi$ as well. On the other hand, $u_{i}-\theta \in$ $W_{0}^{1, p_{i}}(\Omega)$ and Lemma 3.25 together with (4.6) and (4.7) implies that $\bar{u}-\theta \in$ $W_{0}^{1, p_{0}}(\Omega)$. Thus we have shown that $\bar{u} \in \mathcal{K}_{\psi, \theta}^{p_{0}}$.

Next we shall show that

$$
\nabla u_{i} \rightarrow \nabla \bar{u} \quad \text { a.e. }
$$

Together with the weak convergence and (4.7) this implies that

$$
u_{i} \rightarrow \bar{u} \quad \text { in } W^{1, t^{\prime}}(\Omega)
$$


for each $t^{\prime}<t$. Thus a small adjustment of $t$ gives

$$
u_{i} \rightarrow \bar{u} \quad \text { in } W^{1 . t}(\Omega)
$$

where $t$ depends only on $n, p_{0}, \alpha, \beta$ and $\mu$.

To prove (4.8) we use a refinement of the method introduced by Kilpeläinen and Malý $[\mathrm{KilM}]$. Since $u_{i}, i=1,2, \ldots$, is the solution to an obstacle problem, it is also an $A_{i}$-supersolution, i.e.

$$
\int_{\Omega} A_{i}\left(x, \nabla u_{i}\right) \cdot \nabla \varphi d x \geq 0
$$

for each $\varphi \in C_{0}^{\infty}(\Omega), \varphi \geq 0$. Choose subdomains $G \Subset G^{\prime} \Subset \Omega$ and let $\varepsilon>0$. Write

$$
E_{i}^{\varepsilon}=\left\{x \in G^{\prime}:\left(A_{i}\left(x, \nabla u_{i}\right)-A_{i}(x, \nabla \bar{u}) \cdot\left(\nabla u_{i}-\nabla \bar{u}\right)>\varepsilon\right\}\right.
$$

for $i=1,2, \ldots$. Pick a cut off function $\eta \in C_{0}^{\infty}\left(G^{\prime}\right)$ such that $0 \leq \eta \leq 1$ and $\eta=1$ on $G$. Set

$$
w_{i}=\min \left(\left(\bar{u}+\varepsilon^{2}-u_{i}\right)^{+}, 2 \varepsilon^{2}\right)
$$

and note that the function $\eta w_{i}$ is nonnegative and $\eta w_{i} \in W_{0}^{1, t}\left(G^{\prime}\right) \subset W_{0}^{1, p_{i}}\left(G^{\prime}\right)$. Hence we can use $\eta w_{i}$ as a test function in (4.11) and this yields

$$
\begin{aligned}
& \int_{G^{\prime} \cap\left\{\left|u_{i}-\bar{u}\right|<\varepsilon^{2}\right\}} A_{i}\left(x, \nabla u_{i}\right) \cdot\left(\nabla u_{i}-\nabla \bar{u}\right) \eta d x \\
& \quad \leq \int_{G^{\prime}} A_{i}\left(x, \nabla u_{i}\right) \cdot \nabla \eta w_{i} d x \leq C \varepsilon^{2} \int_{G^{\prime}}\left|A_{i}\left(x, \nabla u_{i}\right)\right| d x \\
& \quad \leq C \varepsilon^{2} \int_{G^{\prime}}\left|\nabla u_{i}\right|^{p_{i}-1} d x \leq C \varepsilon^{2}\left(\int_{\Omega}\left|\nabla u_{i}\right|^{p_{i}} d x\right)^{\left(p_{i}-1\right) / p_{i}}|\Omega|^{1 / p_{i}} \\
& \leq C \varepsilon^{2}
\end{aligned}
$$

where $C<\infty$ is independent of $i=1,2, \ldots$ 
On the other hand,

$$
\begin{aligned}
\frac{1}{\varepsilon} \int_{G^{\prime} \cap\left\{\left|u_{i}-\bar{u}\right|<\varepsilon^{2}\right\}}\left(A_{i}\left(x, \nabla u_{i}\right)-A_{i}(x, \nabla \bar{u})\right) \cdot\left(\nabla u_{i}-\nabla \bar{u}\right) \eta d x \\
=\frac{1}{\varepsilon} \int_{G^{\prime} \cap\left\{\left|u_{i}-\bar{u}\right|<\varepsilon^{2}\right\}} A_{i}\left(x, \nabla u_{i}\right) \cdot\left(\nabla u_{i}-\nabla \bar{u}\right) \eta d x \\
\quad-\frac{1}{\varepsilon} \int_{G^{\prime} \cap\left\{\left|u_{i}-\bar{u}\right|<\varepsilon^{2}\right\}}\left(A_{i}(x, \nabla \bar{u})-A_{0}(x, \nabla \bar{u})\right) \cdot\left(\nabla u_{i}-\nabla \bar{u}\right) \eta d x \\
\quad-\frac{1}{\varepsilon} \int_{G^{\prime} \cap\left\{\left|u_{i}-\bar{u}\right|<\varepsilon^{2}\right\}} A_{0}(x, \nabla \bar{u}) \cdot\left(\nabla u_{i}-\nabla \bar{u}\right) \eta d x \\
=I_{1}^{i}+I_{2}^{i}+I_{3}^{i}
\end{aligned}
$$

and we have from (4.12) that

$$
I_{1}^{i} \leq C \varepsilon
$$

The second integral can be estimated as follows:

$$
\begin{aligned}
\left|I_{2}^{i}\right| & \leq \frac{1}{\varepsilon}\left\|A_{i}(x, \nabla \bar{u})-A_{0}(x, \nabla \bar{u})\right\|_{p_{i}^{\prime}}\left\|\nabla \bar{u}-\nabla u_{i}\right\|_{p_{i}} \\
& \leq \frac{C}{\varepsilon}\left\|A_{i}(x, \nabla \bar{u})-A_{0}(x, \nabla \bar{u})\right\|_{p_{i}^{\prime}}
\end{aligned}
$$

where $p_{i}^{\prime}$ is the conjugate exponent of $p_{i}$. We show that

$$
\int_{\Omega}\left|A_{i}(x, \nabla \bar{u})-\mathcal{A}_{0}(x, \nabla \bar{u})\right|^{p_{i}^{\prime}} d x \rightarrow 0
$$

as $i \rightarrow \infty$. Now by the assumption (2.4)

$$
A_{i}(x, \nabla \bar{u}(x)) \rightarrow A_{0}(x, \nabla \bar{u}(x))
$$

a.e. in $\Omega$ and for any $E \subset \Omega$

$$
\begin{aligned}
\int_{E}\left|\mathcal{A}_{i}(x, \nabla \bar{u})-\mathcal{A}_{0}(x, \nabla \bar{u})\right|^{p_{i}^{\prime}} d x \\
\leq C\left[\int_{E}|\nabla \bar{u}|^{p_{i}} d x+\int_{E}|\nabla \bar{u}|^{\left(p_{0}-1\right) p_{i}^{\prime}} d x\right] \\
\leq C\left[|E|^{1-\frac{p_{i}}{t}}\left(\int_{E}|\nabla \bar{u}|^{t} d x\right)^{p_{i} / t}+|E|^{1-\left(p_{0}-1\right) p_{i}^{\prime} / t}\left(\int_{E}|\nabla \bar{u}|^{t} d x\right)^{\left(p_{0}-1\right) p_{i}^{2} / t}\right]
\end{aligned}
$$


where $C<\infty$ is independent of $i$. The right side can be made less than $\eta>0$ if $|E|<\delta_{\eta}$. The Vitali convergence theorem now implies (4.16) and from (4.15) we obtain the estimate

$$
\left|I_{2}^{i}\right|<\varepsilon
$$

provided that $i$ is large enough.

For the last integral we set

$$
M_{i}=\left\{x \in G^{\prime}:\left|u_{i}(x)-\bar{u}(x)\right|<\varepsilon^{2}\right\} .
$$

Let $\chi_{i}$ and $\chi_{G^{\prime}}$ be the characteristic functions of $M_{i}$ and $G^{\prime}$, respectively. Now

$$
\begin{aligned}
I_{3}^{i}= & \frac{1}{\varepsilon} \int_{G^{\prime}} \chi_{i} A_{0}(x, \nabla \bar{u}) \cdot\left(\nabla u_{i}-\nabla \bar{u}\right) \eta d x \\
= & \frac{1}{\varepsilon} \int_{G^{\prime}}\left(\chi_{i}-\chi_{G^{\prime}}\right) A_{0}(x, \nabla \bar{u}) \cdot\left(\nabla u_{i}-\nabla \bar{u}\right) \eta d x \\
& \quad+\frac{1}{\varepsilon} \int_{\Omega} \chi_{G^{\prime}} A_{0}(x, \nabla \bar{u}) \cdot\left(\nabla u_{i}-\nabla \bar{u}\right) \eta d x
\end{aligned}
$$

and since $\nabla u_{i} \rightarrow \nabla u$ weakly in $L^{t}(\Omega)$, and hence weakly in $L^{p_{0}}(\Omega)$, we have

$$
\int_{\Omega} \chi_{G^{\prime}} A_{0}(x, \nabla \bar{u}) \cdot\left(\nabla u_{i}-\nabla \bar{u}\right) \eta d x \rightarrow 0
$$

as $i \rightarrow \infty$. For the first integral observe that

$$
\left|\left(\chi_{i}-\chi_{G^{\prime}}\right) A_{0}(x, \nabla \bar{u})\right| \leq 2\left|A_{0}(x, \nabla \bar{u})\right| \in L^{p_{0}^{\prime}}\left(G^{\prime}\right)
$$

and since $u_{i} \rightarrow u$ a.e. in $G^{\prime}$,

$$
\left(\chi_{i}-\chi_{G^{\prime}}\right) A_{0}(x, \nabla \bar{u}) \rightarrow 0 \quad \text { a.e. in } G^{\prime}
$$

Thus the Lebesgue dominated convergence theorem and the Hölder inequality imply that the first integral converges to zero as $i \rightarrow 0$. Consequently we have

$$
\left|I_{3}^{i}\right|<\varepsilon
$$

for large $i$.

Combining (4.14), (4.17), and (4.18) we obtain for large $i$

$$
\frac{1}{\varepsilon} \int_{G^{\prime} \cap\left\{\left|u_{i}-\bar{u}\right|<\varepsilon^{2}\right\}}\left(A_{i}\left(x, \nabla u_{i}\right)-A_{i}(x, \nabla \bar{u})\right) \cdot\left(\nabla u_{i}-\nabla \bar{u}\right) \eta d x \leq C \varepsilon
$$


where $C$ is independent of $i$ and $\varepsilon$. Since $E_{i}^{\varepsilon} \subset G^{\prime}$, the ellipticity assumption (d) yields

$$
\begin{aligned}
\left|E_{i}^{\varepsilon}\right| \leq\left|E_{i}^{\varepsilon} \cap\left\{\left|u_{i}-\bar{u}\right| \geq \varepsilon^{2}\right\}\right| & \\
& \quad+\frac{1}{\varepsilon} \int_{E_{i}^{\varepsilon} \cap\left\{\left|u_{i}-\bar{u}\right|<\varepsilon^{2}\right\}}\left(A_{i}\left(x, \nabla u_{i}\right)-A_{i}(x, \nabla \bar{u})\right) \cdot\left(\nabla u_{i}-\nabla \bar{u}\right) d x \\
& \leq\left|E_{i}^{\varepsilon} \cap\left\{\left|u_{i}-\bar{u}\right| \geq \varepsilon^{2}\right\}\right|+C \varepsilon \\
& \leq C \varepsilon
\end{aligned}
$$

for large $i$, say $i \geq i_{0}$, because $u_{i} \rightarrow \bar{u}$ a.e. Now the ellipticity assumption, together with (4.19), easily shows that

$$
\lim _{i \rightarrow \infty} I_{i}(x)=\lim _{i \rightarrow \infty}\left(A_{i}\left(x, \nabla u_{i}\right)-A_{i}(x, \nabla \bar{u})\right) \cdot\left(\nabla u_{i}-\nabla \bar{u}\right)=0
$$

for a.e. $x \in G^{\prime}$. This is the main step for (4.8).

It suffices to show that (4.8) holds a.e. in $G^{\prime}$. To this end let $x_{0} \in G^{\prime}$ with

(i) $I_{i}\left(x_{0}\right) \rightarrow 0$ as $i \rightarrow \infty$,

(ii) $\left|\nabla \bar{u}\left(x_{0}\right)\right|<\infty$,

(iii) (b)-(d) hold for $x=x_{0}$ and $A=A_{i}, i=0,1, \ldots$, and

(iv) $A_{i}\left(x_{0}, \xi\right) \rightarrow A_{0}\left(x_{0}, \xi\right)$ locally uniformly in $\mathbb{R}^{n}$, see (2.4).

If $\left|\nabla u_{i}\left(x_{0}\right)\right| \rightarrow \infty$, then

$\alpha\left|\nabla u_{i}\left(x_{0}\right)\right|^{p_{i}}$

$$
\begin{aligned}
\leq & A_{i}\left(x_{0}, \nabla u_{i}\left(x_{0}\right)\right) \cdot \nabla u_{i}\left(x_{0}\right) \\
= & \left(A_{i}\left(x_{0}, \nabla u_{i}\left(x_{0}\right)\right)-A_{i}\left(x_{0}, \nabla \bar{u}\left(x_{0}\right)\right)\right) \cdot\left(\nabla u_{i}\left(x_{0}\right)-\nabla \bar{u}\left(x_{0}\right)\right) \\
& \quad+A_{i}\left(x_{0}, \nabla \bar{u}\left(x_{0}\right)\right) \cdot\left(\nabla u_{i}\left(x_{0}\right)-\nabla \bar{u}\left(x_{0}\right)\right)+A_{i}\left(x_{0}, \nabla u_{i}\left(x_{0}\right)\right) \cdot \nabla \bar{u}\left(x_{0}\right) \\
\leq & I_{i}\left(x_{0}\right)+\beta\left|\nabla \bar{u}\left(x_{0}\right)\right|^{p_{i}-1}\left(\left|\nabla u_{i}\left(x_{0}\right)\right|+\left|\nabla \bar{u}\left(x_{0}\right)\right|\right)+\beta\left|\nabla u_{i}\left(x_{0}\right)\right|^{p_{i}-1}\left|\nabla \bar{u}\left(x_{0}\right)\right|,
\end{aligned}
$$

and this is a contradiction as $i \rightarrow \infty$. Thus we may assume, passing to a subsequence if necessary, that

$$
\nabla u_{i}\left(x_{0}\right) \rightarrow \xi \in \mathbb{R}^{n}
$$

If now $\xi \neq \nabla \bar{u}\left(x_{0}\right)$, then

$$
0=\lim _{i \rightarrow \infty} I_{i}\left(x_{0}\right)=\left(A_{0}\left(x_{0}, \xi\right)-A_{0}\left(x_{0}, \nabla \bar{u}\left(x_{0}\right)\right) \cdot\left(\xi-\nabla \bar{u}\left(x_{0}\right)\right)>0\right.
$$


provides a contradiction. Since a.e. point $x_{0} \in G^{\prime}$ satisfies (i)-(iv), we have proved that $\nabla u_{i} \rightarrow \nabla u_{0}$ a.e. in $G^{\prime}$ and hence a.e. in $\Omega$. This completes the proof for (4.8) and (4.9) also follows.

At the final step of the proof we will show that

$$
u_{0}=\bar{u}
$$

We know that $\bar{u} \in \mathcal{K}_{\psi, \theta}^{p_{0}}$ and hence

$$
\int_{\Omega} A_{0}\left(x, \nabla u_{0}\right) \cdot\left(\nabla \bar{u}-\nabla u_{0}\right) d x \geq 0 .
$$

In order to obtain the inequality

$$
\int_{\Omega} A_{0}(x, \nabla \bar{u}) \cdot\left(\nabla u_{0}-\nabla \bar{u}\right) d x \geq 0
$$

we use an approximation method; the difficulty is that we do not know that $u_{0}-\theta \in W_{0}^{1, t}(\Omega)$ although $u_{0}, \theta \in W^{1, t}(\Omega)$.

For (4.22) we may again assume that $\theta \geq \psi$. Now $u_{0}-\theta \in W_{0}^{1, p_{0}}(\Omega)$ and hence there is a sequence $\varphi_{i} \in C_{0}^{\infty}(\Omega)$ such that $\varphi_{i} \rightarrow u_{0}-\theta$ in $W^{1, p_{0}}(\Omega)$. Since $\psi-\theta \leq 0$ and since $\varphi_{i}$ has compact support,

$$
\max \left(\varphi_{i}, \psi-\theta\right) \in W_{0}^{1, t}(\Omega)
$$

and then $v_{i}-\theta \in W_{0}^{1, t}(\Omega)$ where $v_{i}=\max \left(\varphi_{i}, \psi-\theta\right)+\theta$. On the other hand

$$
v_{i} \geq \psi-\theta+\theta=\psi \text { a.e. }
$$

and thus $v_{i} \in \mathcal{K}_{\psi, \theta}^{t}$. If $v \in \mathcal{K}_{\psi, \theta}^{t}$, then we may assume that $v \in \mathcal{K}_{\psi, \theta}^{p_{i}}$ for all $i=0$, $1, \ldots$ Hence

$$
\int_{\Omega} A_{i}\left(x, \nabla u_{i}\right) \cdot\left(\nabla v-\nabla u_{i}\right) d x \geq 0
$$

and letting $i \rightarrow \infty$ we obtain from (4.10)

$$
\int_{\Omega} A_{0}(x, \nabla \bar{u}) \cdot(\nabla v-\nabla \bar{u}) d x \geq 0 .
$$

Set $v=v_{i}$ in (4.23). Now $u_{0} \geq \psi$ a.e. and hence $v_{i} \rightarrow u_{0}$ in $W^{1, p_{0}}(\Omega)$. Thus (4.23) implies (4.22).

It is now easy to complete the proof for (4.20). From (d) and from (4.21) and (4.22) we obtain

$$
0 \leq \int_{\Omega}\left(A_{0}(x, \nabla \bar{u})-A_{0}\left(x, \nabla u_{0}\right)\right) \cdot\left(\nabla \bar{u}-\nabla u_{0}\right) d x \leq 0
$$


and this is possible only if (4.20) holds.

The proof for Theorem 2.5 is complete.

\section{REFERENCES}

[AH] D. R. Adams \& L. I. Hedberg, Function Spaces and Potential Theory, Springer, 1996.

[G] M. Giaquinta, Remarks on the regularity of weak solutions of some variational inequalities, Math. Z. 177 (1981), 15-33.

[GT] D. Gilbarg 63 N. S. Trudinger, Elliptic Partial Differential Equations of Second Order, 2nd Edition, Springer-Verlag, 1983.

[HKM] J. Heinonen, T. Kilpeläinen 8 O. Martio, Nonlinear Potential Theory of Degenerate Elliptic Equations, Clarendon Press, 1993.

[I] T. Iwaniec, The Gehring Lemma, Quasiconformal Mappings and Analysis: A collection of papers honoring Frederick W. Gehring on his 70th birthday (P. L. Duren et al., eds.), Proceedings of the International Symposium, Ann Arbor, August 1995, Springer-Verlag, 1998, pp. 181-204.

[KilM] T.K̈ILPELÄInen \& J. MALÝ, Degenerate elliptic equations with measure data and nonlinear potentials, Annali della Scuola Normala Superiore di Pisa, Ser. IV XIX (1992), 591-613.

[KinM] J. Kinnunen $\mathcal{G}$ O. Martio, Hardy's inequalities for Sobolev functions, Math. Research Lett. 4 (1997), 489-500.

[Le] J. L. Lewis, Uniformly fat sets, Trans. Amer. Math. Soc. 308 (1988), 177-196.

[Li] P. Lindqvist, Stability for the solutions of $\operatorname{div}\left(|\nabla u|^{p-2} \nabla u\right)=f$ with varying $p$, J. Math. Anal. Appl. 127 (1987), 93-102.

[LU] O. A. Ladyzenskaya 8 N. N. Uraltseva, Linear and Quasilinear Equations of Elliptic Type, Russian, Nauka, 1964.

[LM1] Li Gongbao 83 O. Martio, Local and global integrability of gradients in obstacle problems, Ann. Acad. Sci. Fenn. Ser. A I Math. 19 (1994), 25-34.

[LM2] - Stability in obstacle problems, Math. Scand. 75 (1994), 87-100.

$[\mathrm{M}]$ P. MikKonen, On the Wolff potential and quasilinear elliptic equations involving measures, Ann. Acad. Sci. Fenn. Ser. A I Math. Diss. 104 (1996).

[Z] W. P. Ziemer, Weakly Differentiable Functions: Sobolev Spaces and Functions of Bounded Variation, Springer, 1989.

Acknowledgment. The first author was partially supported by the Academy of Finland and NSFC.

\section{Li Gongbao}

Young Scientist Lab. of Math. Physics

Wuhan Institute of Physics and Mathematics

Chinese Academy of Sciences

P.O. Box 71010, Wuhan 430071

Peoples' Republic of China
Olli Martio

P.O. Box 4

Department of Mathematics

University of Helsinki

FIN-00014 University of Helsinki

Finland

EMAIL: olli.martio@helsinki.fi

Received: June 5th, 1997; revised: October 2nd, $199 \%$. 